\title{
On Reduced Order Modeling of Flexible Structures from Frequency Response Data
}

\author{
Okan Demir ${ }^{1}$ and Hitay Özbay ${ }^{1}$ \\ ${ }^{1}$ Dept. of Electrical \& Electronics Engineering, Bilkent University, Ankara 06800 Turkey
}

\begin{abstract}
In order to identify the dominant flexible modes of a flexible structure with an input/output delay, a numerical method is proposed. The method uses a frequency domain approach (frequency response data) to estimate the resonating frequencies and damping coefficients of the flexible modes, as well as the amount of the time delay. A sequential NLLS (NonLinear Least Squares) curve fitting procedure is adopted. It is illustrated that such a Newtonian optimization method has the capability of finding the parameters of a reduced order transfer function by minimizing a cost function involving nonlinearities such as exponential and fractional terms.
\end{abstract}

\section{INTRODUCTION}

One of the challenging tasks in the control of flexible structures is the estimation of the dominant modes (location of resonant frequencies and associated damping coefficients). There are several PDE based models for various types of flexible systems, see e.g. [6]. An attractive feature of these infinite dimensional (spatially distributed) models is that they contain only a few parameters, and modal properties can be computed easily, [14]. On the other hand, small uncertainty in these parameters may lead to large errors in the frequency response, which in turn make any internal model based control scheme unreliable (non-robust). For this reason, in practice, unless material properties of the structure is perfectly known, majority of the identification methods rely on experimentally collected input-output data, [1]. Typically, by applying sinusoidal inputs of varying frequency, the frequency response (magnitude and phase of the transfer function) of the flexible structure is obtained. The main issue in this method is to determine the dominant modes and the amount of time delay in the system (the phase shift due to time delay may be attributed to non-collocated actuators and sensors, but this may also be due to slow reacting actuator dynamics). The present paper deals with this issue by using the available frequency response data. The "true" transfer function is assumed to be in the form $G(s)$ or $G_{o}(s)$ given below:

$$
G(s)=\frac{K_{0}}{s} G_{o}(s) \quad \text { and } \quad G_{o}(s)=P(s) e^{-h s} ;
$$

where $h \geq 0$ is the effective time delay, $P(s)$ is the minimum-phase part, and if the integral action is present $K_{0}$ is the associated gain. The goal is to find estimated values of the parameters $h$ and $K_{0}$ and a reduced order minimum phase transfer function $P_{N}(s)$ approximating $P(s)$.

For many flexible systems when actuators and sensors are collocated, $P(s)$ turns out to be minimum phase. On the other hand, due to non-collocation of the actuators and sensors, the transfer function model may have to contain right half plane zeros. Nevertheless, it is possible to model these non-minimum phase zeros with a single lumped delay parameter, $h>0$, see for example [3], [2].

For a general linear time invariant system, from a discrete time domain data a DFT based estimation of its frequency response can be made, [8]. Here, it will be assumed that the frequency response data is available: $G\left(j \omega_{i}\right)$ at sufficiently large number of frequency points $\omega_{i}, i=1, \ldots, M$. For a class of infinite dimensional systems, [10] proposed an approximation method that can be applied for finding a reduced order model $P_{N}$. However, it cannot be used for simultaneous estimation of $h$ and $P_{N}$; and it may result in a non-minimum phase $P_{N}$. A subspace-based identification method was developed in [16]. This method can handle frequency response data on uniformly or non-uniformly spaced frequency intervals and gives a state space form using balanced realization combined with stochastic methods for dealing with noisy data. Other identification methods include ARMA-based models used on time domain data, [12], [13]. PDE-based models are also considered in the literature, see e.g. [18] for an adaptive estimation method for a flexible beam.

In the present paper $P_{N}(s)$ is restricted to be minimum phase and a nonlinear least squares (NLLS) method is used in order to estimate the parameters of the system. The cost function considered here is penalizing the relative error $\left\|\left(G-G_{N}\right) / G_{N}\right\|$ (note that a bound on the relative error is an important information used in robust controller design, [19]). The approach is then demonstrated on three examples: the first one is the frequency response of a free-free beam transfer function from [14]. The second example is a clamped-free beam model taken from [6]. The third example is a damped version of the free-free rod transfer function taken from [17]. In each case, the frequency response data is generated from the infinite dimensional transfer function and the parameters of a finite dimensional model are estimated using the proposed NLLS method.

The problem definition is given in Section 2. The proposed algorithm can be found in Section 3. Three illustrative examples are given in Section 4, and concluding remarks are made in Section 5. 


\section{PROBLEM DEFINITION}

In this paper a method for extracting a finite order model followed by a delay term, that approximates an infinite dimensional transfer function is investigated. The process can be considered in basic terms as a complex curve fitting approach, where the complex valued data is the set of frequency responses $\Phi_{i}:=G\left(j \omega_{i}\right)$ at a specific set of frequency points $\omega_{i}, i=1, \ldots, M$, where $G(s)$ is in the form (1).

The approximation $G_{N} \cong G$, or $G_{N} \cong G_{o}$ is defined as

$$
\begin{aligned}
G_{N}(s) & =e^{-h s} \frac{K_{0}}{s} P_{N}(s) \quad \text { or } \quad G_{N}(s)=e^{-h s} P_{N}(s) \\
P_{N}(s) & =\prod_{k=1}^{N} \frac{\left(a_{k} s^{2}+2 \zeta_{k, n} \omega_{k, n} s+\omega_{k, n}^{2}\right)}{\left(b_{k} s^{2}+2 \zeta_{k, d} \omega_{k, d} s+\omega_{k, d}^{2}\right)} .
\end{aligned}
$$

The terms $a_{k}, b_{k}$ are added in order to adjust the low frequency gain of $P_{N}$.

The objective is to solve an optimization problem to minimize the relative error between $\Phi_{i}$ and $G_{N}\left(j \omega_{i}\right)$. More precisely, the constrained optimization problem is defined as

$$
\begin{aligned}
& \underset{\underline{\beta}}{\operatorname{minimize}} \epsilon(\underline{\beta})=\sum_{i=1}^{M}\left|\frac{\Phi_{i}-G_{N}\left(j \omega_{i}, \underline{\beta}\right)}{G_{N}\left(j \omega_{i}, \underline{\beta}\right)}\right|^{2} \\
& \text { subject to } \underline{\beta} \succeq 0,
\end{aligned}
$$

where ' $\succeq$ ' means element-wise inequality of a vector, and the parameter vector $\underline{\beta}$ is defined as

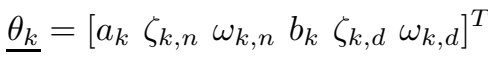

$$
\begin{aligned}
& \underline{\beta}=\left[h, K_{0},{\underline{\theta_{1}}}^{T},{\underline{\theta_{2}}}^{T}, \ldots,{\underline{\theta_{N}}}^{T}\right]^{T}
\end{aligned}
$$

The constrained optimization problem given in (4) is non-linear since it contains a ratio of polynomials and an exponential term. Also the solution must obey the nonnegativity constraint. In order to minimize objective cost function step by step until the relative error becomes sufficiently small, a sequential NLLS approach is preferred. Although NLLS method is slower according to ordinary least squares methods, it gives better results than linearizing the optimization problem (4).

\section{NLLS APPROACH AND PROPOSED ALGORITHM}

\section{A. Gauss-Newton Method}

In terms of a simplified notation the optimization problem defined above can be considered as minimizing

$$
\begin{aligned}
\epsilon(\underline{\beta}) & =\underline{F}^{H} \underline{F} \text { where } \\
\underline{F} & =\left[\begin{array}{c}
\frac{\Phi_{1}-G_{N}\left(j \omega_{1}, \underline{\beta}\right)}{G_{N}\left(j \omega_{1}, \underline{\underline{\beta}}\right)} \\
\frac{\Phi_{2}-G_{N}\left(j \omega_{2}, \underline{\beta}\right)}{G_{N}\left(j \omega_{2}, \underline{\beta}\right)} \\
\vdots \\
\frac{\Phi_{M}-G_{N}\left(j \omega_{M}, \underline{\beta}\right)}{G_{N}\left(j \omega_{M}, \underline{\beta}\right)}
\end{array}\right]=\left[\begin{array}{c}
F_{1} \\
F_{2} \\
\vdots \\
F_{M}
\end{array}\right]
\end{aligned}
$$

For solving a set of non-linear equations, Gauss-Newton method is preferable. Although Gauss-Newton method can not guarantee convergence to the global minimum, it is certain that it iterates through a local minimum [7]. However, for good convergence properties, initial values in the parameter space must be selected carefully. There is no perfectly defined initialization point, it can be selected in a logical way for our specific case.

Let $\beta=\beta_{c}$ denote the current selection of the parameters, they are updated using a Newton-step modified by a damping term of the Levenberg-Marquardt method. Modified solution of the Newton-step is defined as;

$$
\begin{aligned}
M_{L M} & =J^{H}\left(\underline{\beta_{c}}\right) J\left(\underline{\beta_{c}}\right)+\sigma I, \\
\underline{s_{N}} & =-M_{L M}^{-1}\left(\underline{\beta_{c}}\right) J^{H}\left(\underline{\beta_{c}}\right) \underline{F}\left(\underline{\beta_{c}}\right), \\
\underline{\beta_{+}} & =\underline{\beta_{c}}+\underline{s_{N}},
\end{aligned}
$$

where $I$ is the identity matrix and $\sigma$ is the damping coefficient [15].

So far unconstrained optimization is discussed. Further, non-negativity constraints should also be handled, since all poles and zeros of $G_{N}(s)$ are restricted to be in the left half plane when integrator and delay terms are removed. A simple solution is to use barrier functions, [4]. Logarithmic barrier function is used in our case. $\underline{F}$ in the modified objective function is

$$
\begin{gathered}
\underline{F}=\left[\begin{array}{c}
\frac{1}{\mu} \frac{\Phi_{1}-G_{N}\left(j \omega_{1}, \underline{\beta}\right)}{G_{N}\left(j \omega_{1}, \underline{\beta}\right)}-Q(\underline{\beta}) \\
\frac{1}{\mu} \frac{\Phi_{2}-G_{N}\left(j \omega_{2}, \underline{\beta}\right)}{G_{N}\left(j \omega_{2}, \underline{\beta}\right)}-Q(\underline{\beta}) \\
\vdots \\
\frac{1}{\mu} \frac{\Phi_{M}-G_{N}\left(j \omega_{M}, \underline{\beta}\right)}{G_{N}\left(j \omega_{M}, \underline{\beta}\right)}-Q(\underline{\beta})
\end{array}\right], \\
Q(\underline{\beta})=\sum_{i=1}^{K} \log \left(\beta_{i}\right),
\end{gathered}
$$

for some $\mu \in \mathbb{R}, \mu>0$, and $\underline{\beta}$ as defined in (6) has $K$ elements. Furthermore the Jacobian is modified as

$$
J_{i j}(\underline{\beta})=\left[\frac{1}{\mu} \frac{\partial F_{i}(\underline{\beta})}{\partial \beta_{j}}-\frac{1}{\beta_{j}}\right]
$$

Log-barrier method has similarities with solution methods for dual optimization problems and efficient for most cases. The above discussion summarizes the main idea behind the steps of the algorithm which is described below.

\section{B. The algorithm}

Complex curve fitting operation is made in arbitrary intervals on the frequency domain. By starting from lowest frequency data point, an iterative parameter search sub-step is performed by taking the frequency point, where the highest error between $\Phi$ and $G_{N}(s)$ occurs, as the initialization point. Outer loop is continued until reaching a reasonable error level or a given transfer function order.

Applying NLLS methods on all data points at one outer step makes it difficult to define model order and initial values of parameters and will not give desired result. Therefore, selection of frequency intervals where resonant/anti-resonant peaks are visible gives a good starting point. An admissible approach for data point selection is starting from the lowest 
frequency region and working our way to high frequencies we try to estimate the values of resonance frequencies and damping coefficients by successively adding the frequency intervals where the highest relative error occurs to the previously considered intervals.

These intervals are defined as:

$$
\begin{aligned}
& A=\left\{A_{L_{0}}, A_{L_{1}}, \ldots A_{L_{N}} \mid 1 \leq L_{i} \leq M\right\}, \\
& A_{L_{i}}=\left\{\Phi_{l} \mid l=1,2, \ldots L_{i}\right\}, \\
& L_{i+1}=\max \left\{\underset{i}{\arg }\left\|\frac{\Phi_{i}-G_{N}\left(j \omega_{i}\right)}{G_{N}\left(j \omega_{i}\right)}\right\|_{\infty}, L_{i}\right\} \text {, } \\
& A_{L_{0}} \subseteq A_{L_{1}} \subseteq \cdots \subseteq A_{L_{N}}, \\
& B=\left\{B_{L_{0}}, B_{L_{1}}, \ldots B_{L_{N}} \mid 1 \leq L_{i} \leq M\right\}, \\
& B_{L_{i}}=\left\{j \omega_{l} \mid l=1,2, \ldots L_{i}\right\}, \\
& B_{L_{0}} \subseteq B_{L_{1}} \subseteq \cdots \subseteq B_{L_{N}} .
\end{aligned}
$$

Step 1 Set $t o l \in \mathbb{R}^{+}$a very small number and $\mathcal{N}$, where $2 \mathcal{N}-1$ defines the degree of the resulting transfer function $G_{N}(s)$,

Step 2 Set $N \leftarrow 0$

Step 3 Calculate integral gain $K_{0}$;

Step $i$ Define $L_{0} \leftarrow L_{\text {init }}$, where $L_{\text {init }} \in \mathcal{Z}^{+}$and $L_{\text {init }} \ll M$,

Step ii Define $A_{L_{0}}$ and $B_{L_{0}}$, according to (12), (13),

Step iii Define $G_{N}(s), G_{0}(s)=e^{-h s} \frac{K}{s}$,

Step iv Initialize: $K \leftarrow \frac{\left|\Phi_{1}\right|}{w_{1}}$ and $h \leftarrow 0$,

Step $v$ Solve constrained optimization problem (4),

[15], [5],

Step 4 Set $\omega_{c} \leftarrow \underset{\omega_{i}}{\arg }\left\|\frac{\Phi_{i}-G_{N}\left(j \omega_{i}\right)}{G_{N}\left(j \omega_{i}\right)}\right\|_{\infty}$,

Step 5 Calculate the coefficients of increasing number of resonance/anti-resonance terms,

Step $\boldsymbol{i} L_{N+1}=\max \left\{\underset{i}{\arg }\left\|\frac{\Phi_{i}-G_{N}\left(j \omega_{i}\right)}{G_{N}\left(j \omega_{i}\right)}\right\|_{\infty}, L_{N}\right\}$,

Step ii Set $N \leftarrow N+1$,

Step iii Define data sets $A_{L_{N}}$ (12) and $B_{L_{N}}$ (13),

Step iv Define parameter vector $\underline{\beta}=$ $\left[K_{0}, h,{\underline{\theta_{1}}}^{T}, \ldots,{\underline{\theta_{N}}}^{T}\right]^{T}$ as given in (6).

Step $v$ Initialize: $\overline{\theta_{N}}$, select $a_{N}=1, \zeta_{N, n}=$

$0.5, \omega_{N, n}=\omega_{c}, b_{N}=1, \zeta_{N, d}=0.5, \omega_{N, d}=\omega_{c}$, values of the remaining items in vector $\beta$ come from the previous iteration.

Step vi Solve optimization problem given in (4) starting at the given initial values of previous step where $G_{N}(s)$ maps set $B_{L_{i}}$ to an approximation of the set $A_{L_{i}}$ [5], [15], Algorithm 11.1 of [4].

Step 6 goto Step 4 if $(N<\mathcal{N}$ and

$\left.\max \left|\frac{\Phi_{i}-G\left(j \omega_{i}, \underline{\theta}\right)}{G_{N}\left(j \omega_{i}, \underline{\theta}\right)}\right|>t o l\right)$.

Step $7 \underline{\beta}=\left[K_{0}, h,{\underline{\theta_{1}}}^{T},{\underline{\theta_{2}}}^{T}, \ldots,{\underline{\theta_{N}}}^{T}\right]^{T}$ and exit

In order to increase the efficiency of barrier method, initial values of $\theta_{k}$ can be modified, they can be selected as $\theta_{k}=$ $\left[l a_{k}, \sqrt{l} \zeta_{k, n}, \sqrt{l} \omega_{k, n}, l b_{k}, \sqrt{l} \zeta_{k, d}, \sqrt{l} \omega_{k, d}\right]^{T}$, where $l>1$.

Finally after obtaining an $(2 \mathcal{N}-1)$-th order approximate model for the infinite dimensional system, a balance and
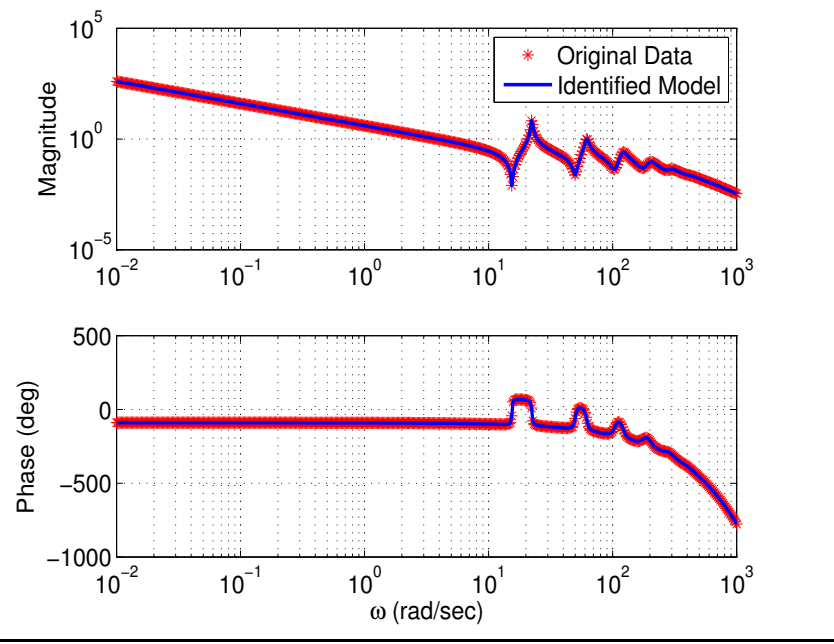

Fig. 1. Bode plots of $G_{A}(s)$ and the identified model $G_{N}(s)$.

truncate method can be used to further reduce the order of the system, [9], [11].

\section{NUMERICAL EXAMPLES}

Three different transfer functions are used for simulation purposes in order to show efficiency of the proposed algorithm. In all the examples $M=500$ and frequency response is available at logarithmically spaced frequency points $\left(\omega_{i}\right)$ between $10^{-2} \mathrm{rad} / \mathrm{sec}$ and $10^{3} \mathrm{rad} / \mathrm{sec}$.

\section{A. Free-Free Beam System}

First example is the transfer function of free-free flexible beam which has an integrator term and a delay term of $h=$ $0.01 \mathrm{sec}$. Its transfer function from force input to velocity measurement is, [14],

$$
G_{A}(s)=\frac{-s e^{-h s}}{\left(\epsilon_{1} s+1\right)\left(\epsilon_{2} s+1\right)} \frac{(\sinh \beta \cos \beta-\cosh \beta \sin \beta)}{\beta^{3}(\cos \beta \cosh \beta-1)}
$$

where $\beta^{4}=\frac{-s^{2}}{\left(1+\epsilon_{1} s\right)}$ and the damping parameters are selected as $\epsilon_{1}=0.001$, and $\epsilon_{2}=0.0033$.

By applying the algorithm given in above, a 34th order $P_{N}(s)$ is determined; the estimated delay value is $h=$ $0.0106 \mathrm{sec}$. The Bode plots of the original transfer function and that of $G_{N}(s)$ are given in Figure 1; the resulting relative error is as shown in Figure 2. The pole-zero map of $G_{N}$ is shown in Figure 3, see Figure 4 for detailed view. The Hankel singular values of $P_{N}$ are as shown in 5 .

\section{B. Clamped-Free Beam}

An infinite dimensional transfer function of a vibrating beam determined in [6] is taken into consideration as a second example. The transfer function is defined as

$$
G_{B}(s)=\frac{N(s)}{E \operatorname{Im}^{3}(s) D(s)}
$$




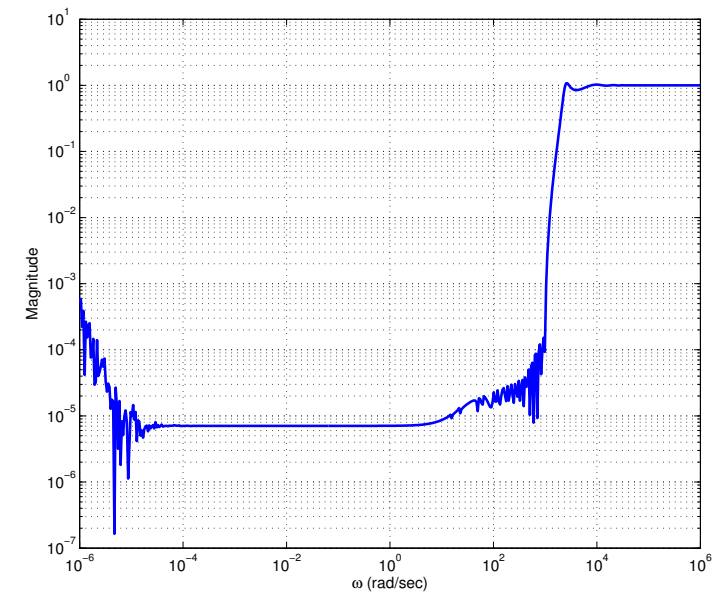

Fig. 2. Relative error $\left|G_{A}(j \omega)-G_{N}(j \omega)\right| /\left|G_{N}(j \omega)\right|$.

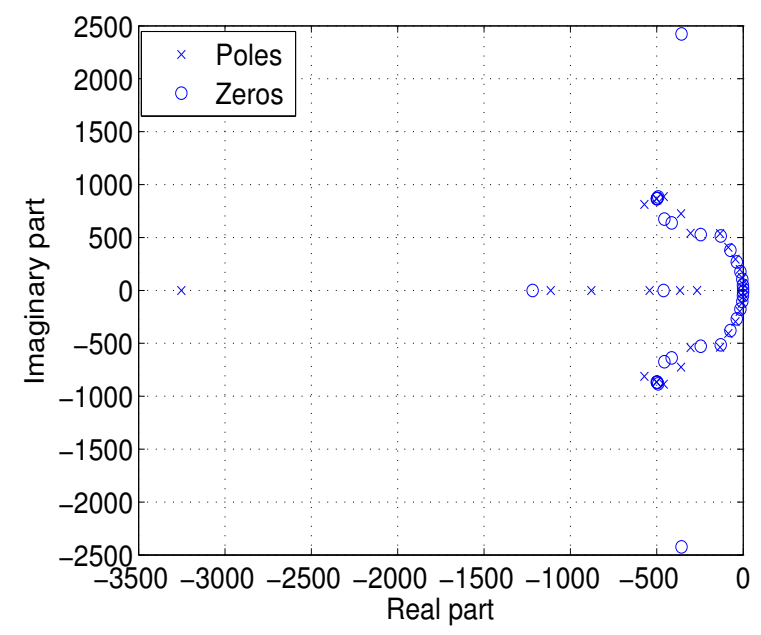

Fig. 3. Pole zero map of $G_{N}$.

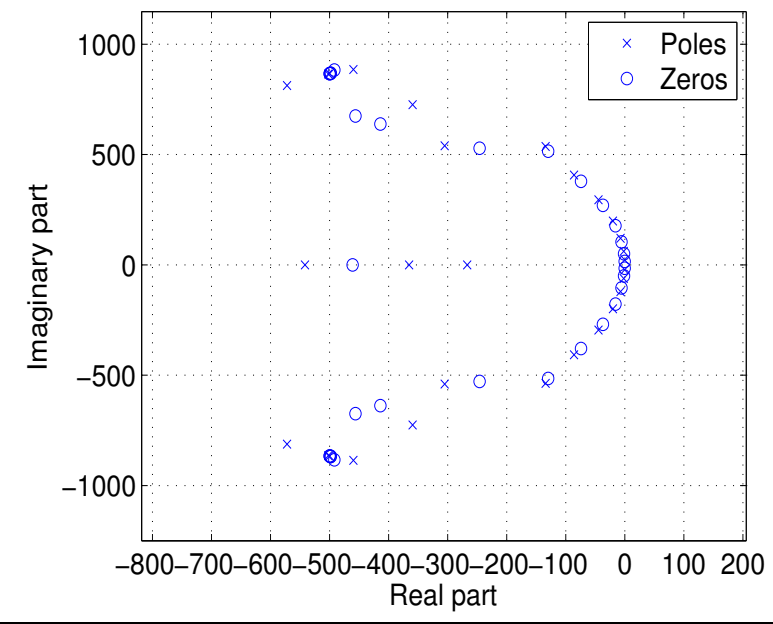

Fig. 4. Zoomed pole zero map of $G_{N}$.

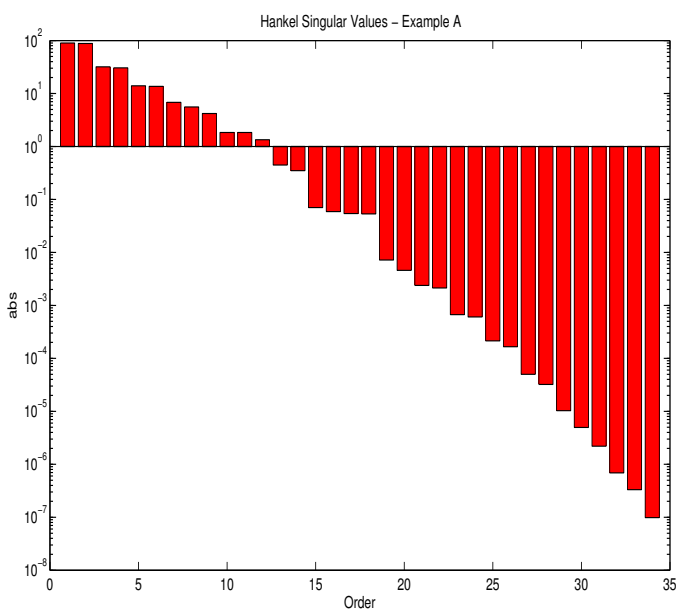

Fig. 5. Hankel singular values of $P_{N}(s)$ for example A.

where

$$
\begin{gathered}
m(s)=\left(\frac{-s^{2}}{E I+s c_{d} I}\right)^{1 / 4} \\
N(s)=\cosh (L m(s)) \sin (L m(s))-\sinh (L m(s)) \cos (L m(s)), \\
D(s)=1+\cosh (L m(s)) \cos (L m(s)) .
\end{gathered}
$$

Here, $E$ and $I$ are material constants and selected as $E=$ 5 and $I=1 ; c_{d}$ is the damping constant and selected as $c_{d}=0.01$. The beam is clamped at $x=0$ and free at $x=L$ where $L=5.5$.

At first, proposed algorithm is modified in order to make basis functions proper. $G_{N}$ is selected as $G_{N}=\underline{1}\left|\Phi_{1}\right|$ for $N=0$, where 1 is a vector consisting of ones and Step 3 is ignored since $G_{B}(s)$ does not have a pole at the origin. The resulting $G_{N}(s)$ has a time delay term which is $h=$ $0.011 \mathrm{sec}$. The degree of $P_{N}(s)$ is 18 and $G_{N}(s)$ does not contain an integral term. Bode plots of $G_{B}(s)$ and $G_{N}(s)$ are given in Figure 6, and the relative approximation error is shown in Figure 7. The Hankel singular values of $G_{N}$ are shown in Figure 8.

\section{Free-Free Uniform Rod}

The third example is an infinite dimensional transfer function which is a damped version of the wave system studied in [17]. This transfer function is expressed by delay terms as follows:

$$
G_{C}(s)=\frac{c}{G I_{p}} \frac{e^{-2 \tau(1-\beta) s}+1}{(\epsilon s+1)-e^{-2 \tau s}} e^{-\tau \beta s},
$$

where parameters selected as $\beta=0.5, \tau=0.0525, c=0.2$, $G=0.4, I_{p}=2.5$ and $\epsilon=0.001$. Resulting transfer function $P_{N}(s)$ is 54th order. The identified $G_{N}(s)$ contains an integral term and a time delay whose estimated value is 0.0261 , which is very close to the exact value $\tau \beta$. Bode plots of $G_{C}(s)$ and $G_{N}(s)$ are given in Figure 9, and the relative approximation error is shown in Figure 10. The Hankel singular values of $G_{N}$ are shown in Figure 11. 

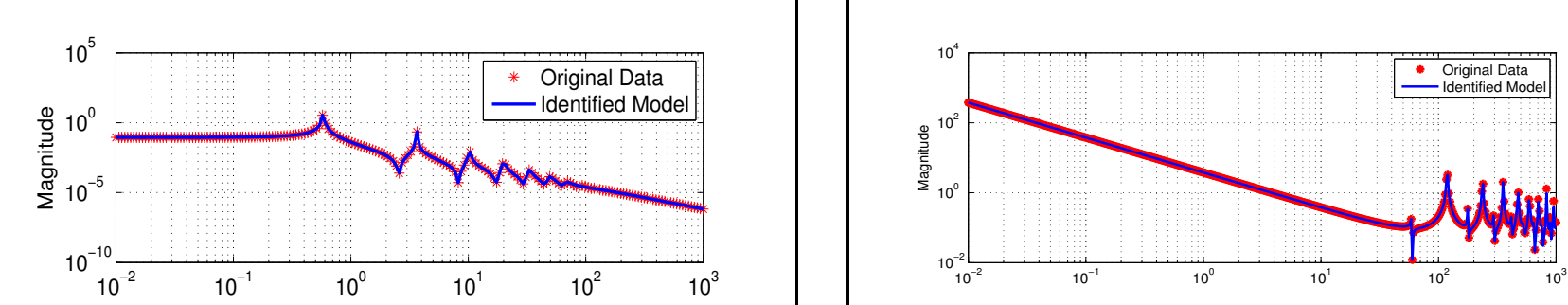

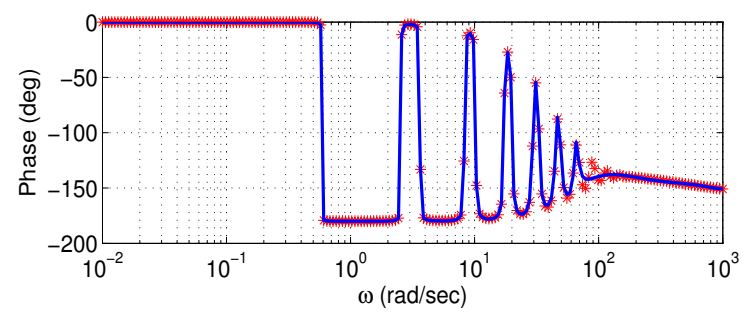

Fig. 6. Bode plots of $G_{B}(s)$ and the identified model $G_{N}(s)$.

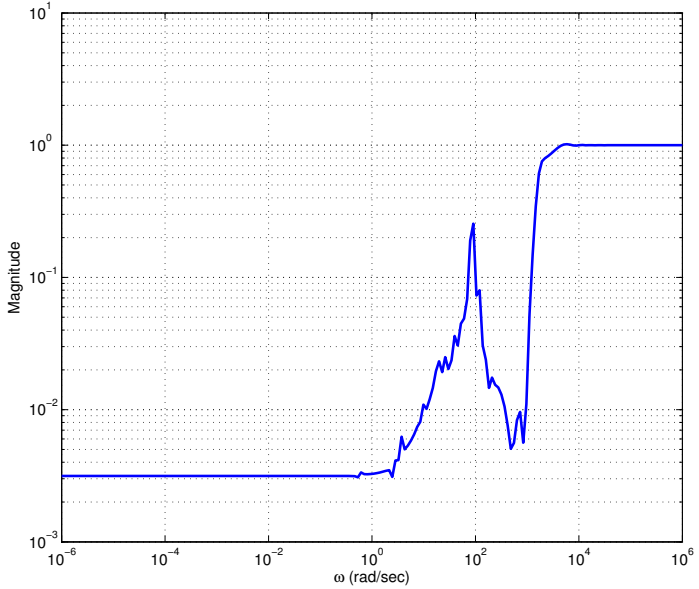

Fig. 7. Relative error $\left|G_{B}(j \omega)-G_{N}(j \omega)\right| /\left|G_{N}(j \omega)\right|$.

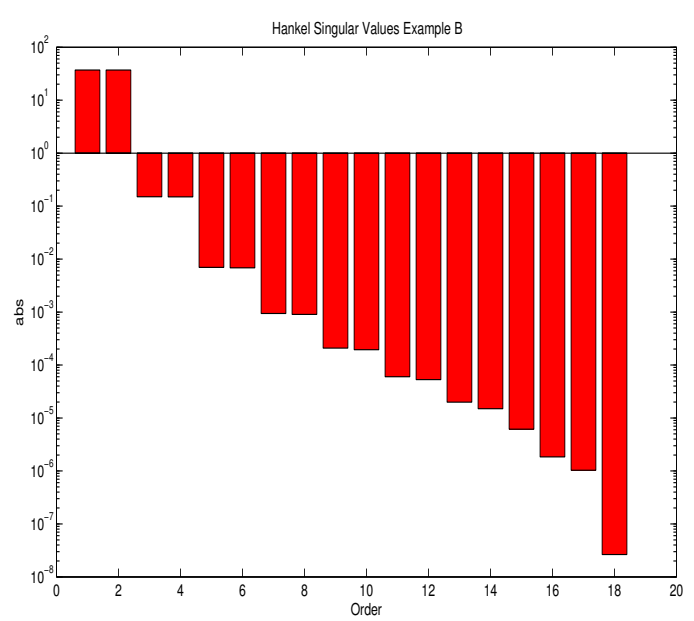

Fig. 8. Hankel singular values of $P_{N}(s)$, for example $B$.

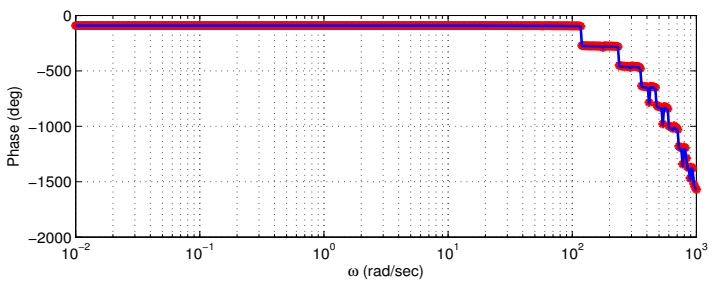

Fig. 9. Bode plots of $G_{C}(s)$ and the identified model $G_{N}(s)$.

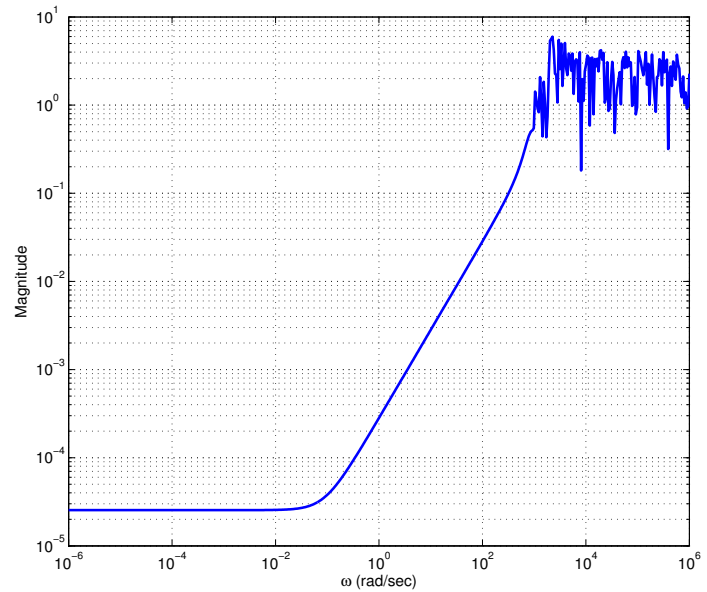

Fig. 10. Relative error $\left|G_{C}(j \omega)-G_{N}(j \omega)\right| /\left|G_{N}(j \omega)\right|$.

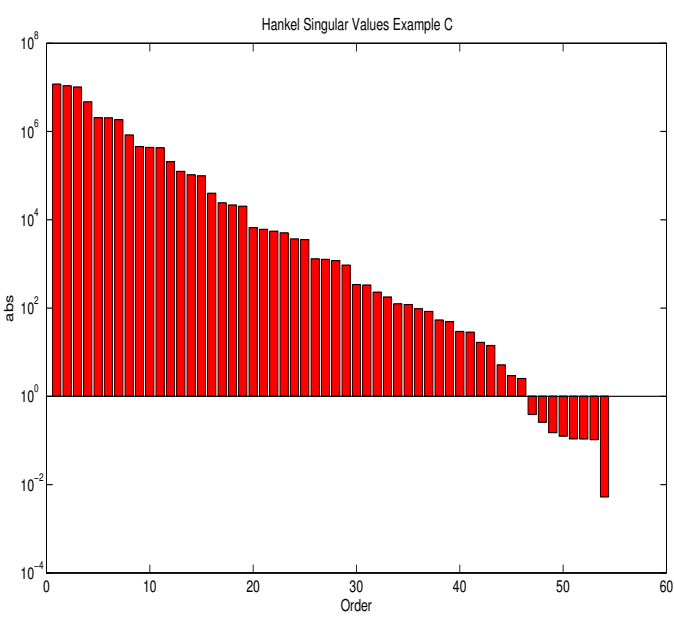

Fig. 11. Hankel singular values of $P_{N}(s)$ for example $C$. 


\section{CONCLUSIONS}

The goal of this paper was to represent an infinite dimensional transfer function by a finite dimensional model and a delay term. For this purpose a NLLS approach is proposed to minimize a cost function which is quadratic in relative error in the frequency response. In order to enhance the results a version of Levenberg-Marquardt algorithm is used and a log-barrier is added to the cost function since pole and zero locations have constraints that they must be in the left-halfplane. As future work, improvements on barrier functions are to be made in order to handle the objective optimization problem's ill-condition for some cases where the damping coefficients are very small.

The results are illustrated with three different examples. From the relative error plots it is observed that the modeling is very good within the frequency range where frequency response data is taken (for all three examples this was between $10^{-2} \mathrm{rad} / \mathrm{sec}$ and $\left.10^{3} \mathrm{rad} / \mathrm{sec}\right)$. However, the relative error becomes large outside this frequency band. Also, the Hankel singular value plots show that the order of $P_{N}(s)$ can be further reduced. Nevertheless, having a large number poles and zeros close to the imaginary axis forces $P_{N}$ to be of relatively large degree.

\section{REFERENCES}

[1] G. Balas, J. Doyle, "Identification of flexible structures for robust control”, IEEE Control Systems Magazine, vol. 10 (1990), pp. 51-58.

[2] M. Karkoub, G. Balas, K. Tamma, M. Donath, "Robust control of flexible manipulators via $\mu$-synthesis," Control Engineering Practice, vol. 8 (2000), pp. 725-734.

[3] D. Enns, H. Özbay, and A. Tannenbaum, "Abstract model and controller design for an unstable aircraft", Journal of guidance, control, and dynamics vol. 15 (1992): pp. 498-508.

[4] S. P. Boyd and L. Vandenberghe, Convex Optimization, Cambridge University Press, 2004.

[5] M. Bydder, "Solution of a Complex Least Squares Problem with Constrained Phase", Linear Algebra Appl, vol. 433 (2010), pp. 17191721.

[6] R. Curtain and K. Morris, "Transfer functions of distributed parameter systems: A tutorial", Automatica, vol. 45 (2009), pp. 1101-1116.

[7] J. J. E. Dennis and R. B. Schnabel, Numerical Methods for Unconstrained Optimization and Nonlinear Equations, SIAM, 1983.

[8] G. F. Franklin, J. D. Powell, and M. Workman, Digital Control of Dynamic Systems, 3rd Ed., Ellis-Kagle Press, 1998.

[9] K. Glover, "All optimal Hankel-norm approximations of linear multivariable systems and their $L^{\infty}$-error bounds", International Journal of Control, vol. 39 (1984), pp. 1115-1193.

[10] G. Gu, P. P. Khargonekar, and E. B. Lee., "Approximation of infinitedimensional systems", IEEE Transactions on Automatic Control, vol. 34 (1989), pp. 610-618.

[11] S. Gugercin and A. C. Antoulas, "A Survey of Model Reduction by Balanced Truncation and Some New Results", International Journal of Control, vol. 77 (2004), pp. 748-766.

[12] R. L. Kosut, "Uncertainty model unfalsification: A system identification paradigm compatible with robust control design", Proc. of the 34th IEEE Conference on Decision and Control, New Orleans, LA, December 1995, pp. 3492-3497.

[13] R. L. Kosut, and B. D.O. Anderson, "Least-squares parameter set estimation for robust control design", Proc. of the 1994 American Control Conference, Baltimore, MD, June 1994, pp. 3002-3006.

[14] K. Lenz and H. Özbay, "Analysis and robust control techniques for an ideal flexible beam", Control and Dynamic Systems, C.T.Leondes Ed., vol. 57 (1993), pp. 369-369.

[15] M. I. Lourakis, A brief description of the Levenberg-Marquardt algorithm implemented by levmar, 2005 (available at googlecode.com)
[16] T. McKelvey, H. Akçay, and L. Ljung, "Subspace-Based Multivariable System Identificationfrom Frequency Response Data", IEEE Trans. on Automatic Control, vol. 41 (1996), pp. 960-979.

[17] N. Raskin and Y. Halevi, "Control of flexible structures governed by the wave equation" Proc. of the 2001 American Control Conference, Arlington, VA, June 2001, pp. 2486-2491.

[18] I. G. Rosen and M. A. Demetriou, "Adaptive Estimation of a Flexible Beam", Proc. of the 1st IEEE Regional Conference on Aerospace Control Systems, May 1993, pp. 815-819.

[19] K. Zhou, J. C. Doyle, K. Glover, Robust and Optimal Control, Prentice-Hall, 1996. 